\title{
Dolor abdominal recurrente psicológico o anatómico secundario a síndrome de Wilkie
}

\author{
Mario Andrés Castillo Wismana, María del Carmen Alvarez Galán ${ }^{b}$, \\ Raimundo Rodriguez-Bobada Aguirre ${ }^{\mathrm{b}}$, Julián Arenas Mosqueda ${ }^{\mathrm{c}}$
}

${ }^{a}$ M.I.R. de $2^{\circ}$ año de Medicina de Familia y Comunitaria. Ciudad Real. España.

${ }^{\mathrm{b}}$ Especialista en Medicina de Familia y Comunitaria. Servicio de urgencias del Hospital General Universitario de Ciudad Real. España.

' Especialista en Medicina de Familia y Comunitaria. Centro de Salud Zona II. Ciudad Real. España.

Correspondencia:

M.A. Castillo Wisman.

Calle Madroños 14,

Ciudad Real 13002

e-mail:

mariocwisman@yahoo.es.

Recibido el 19 de enero de 2014.

Aceptado para su publicación el 17 de marzo de 2014.

\section{RESUMEN}

El síndrome de la arteria mesentérica superior (SAMS) o de Wilkie es una causa inusual de dolor y obstrucción abdominal alta, debido a la compresión de la tercera porción del duodeno, entre la aorta y arteria mesentérica superior. El paciente presenta síntomas como náuseas, vómitos, dolor epigástrico, retraso ponderal o saciedad precoz. Por ser el dolor abdominal un motivo frecuente de consulta en atención primaria y en los servicios de urgencias, presentamos este caso poco frecuente, a tener en cuenta dentro del diagnostico diferencial de un dolor abdominal con síntomas recurrentes y sin respuesta al manejo médico.

A pesar de su rareza, el SAMS es una patología cada vez más vista en nuestra población, debido al aumento de la cirugía traumatológica vertebral, cirugías mayores, alteraciones de la conducta alimentaria, drogas de abuso, quemados, paraplejías e inmovilizados. Puede diagnosticarse desde atención primaria, ya que se trata de pacientes muy demandantes por la poca respuesta al tratamiento medico inicial, si se desconoce la importancia nutricional en el manejo de estos pacientes.

Presentamos su sintomatología, grupos de riesgo, diagnósticos diferenciales y tratamiento.

Palabras Clave. Obstrucción Duodenal. Síndrome de la Arteria Mesentérica Superior. Duodenoyeyunostomia. Pérdida Aguda de Peso.

\section{ABSTRACT}

Recurrent psychological or anatomical abdominal pain secondary to Wilkie's syndrome Superior mesenteric artery (SMA) (or Wilkie's) syndrome is a rare cause for upper abdominal pain and obstruction, due to compression of the third portion of the duodenum, between the aorta and the superior mesenteric artery. The patient presents with symptoms such as nausea, vomiting, epigastric pain, underweight or early satiety. As abdominal pain is a frequent reason for primary care consultations and using emergency services, we present this unusual case, which should be born in mind within the differential diagnosis of abdominal pain with recurrent symptoms with no response to medical management.

In spite of being rare, SMA is an increasingly common pathology within our population, due to the increase in traumatic surgery, major surgery, eating disorders, drug abuse, burns, paraplegias and disabilities. It can be diagnosed from primary care since the patients are very demanding because of the lack of response to initial medical treatment if the nutritional importance in the management of these patients is unknown.

We present its symptomatology, risk groups, differential diagnosis and treatment.

Key words: Duodenal obstruction. Superior mesenteric artery syndrome. Duodenojejunostomy. Acute weight loss.

\section{INTRODUCCIÓN}

El dolor abdominal comprende entre el 5 y el $10 \%$ de las consultas en los servicios de urgencias (SU), dentro del cual el dolor abdominal indiferenciado ocupa el $25 \%$ de los pacientes dados de alta ${ }^{1}$. Es muy importante conocer y detectar las causas de dolor abdominal potencialmente mortales como son: aneurisma de aorta abdominal, isquemia mesentérica, obstrucción intestinal aguda, ruptura esplénica, perforación del tracto gastrointestinal (úlceras, apendicitis), embarazo ectópico, infarto agudo de miocardio y volvulus. 
Presentamos el caso de un paciente con síndrome de la arteria mesentérica superior (SAMS), una entidad poco frecuente de obstrucción intestinal alta, ya descrita por Boernelu y definida como una obstrucción duodenal de etiología poco esclarecida ${ }^{2,3}$. La clínica fue descrita en 1861 por Rokitanski y en 1927 Wilkie publicó una serie de 75 pacientes ${ }^{4}$. Tiene una prevalencia que ronda el $0,3 \%$ en la población general y hasta 2004, solo había publicados 400 casos $^{5}$.

EI SAMS se produce por la compresión de la tercera porción duodenal contra la aorta por parte de la arteria mesentérica superior en su origen, formando un ángulo inferior a los $25^{\circ}$, cuando normalmente es mayor de $30^{\circ}$ (siendo esta medida la más sensible para su diagnóstico), con una distancia entre aorta y arteria mesentérica superior mayor de $10 \mathrm{~mm}$ (entre 10 y $28 \mathrm{~mm}$ ) a nivel de la $3^{\mathrm{a}}$ vértebra lumbar ${ }^{6,7}$. Se ha relacionado con la disminución del panículo adiposo retroperitoneal.

Responde en su presentación aguda al manejo médico, que consiste en corrección de volumen e iones, sonda nasogástrica, analgesia, inhibidores de la bomba de protones y soporte nutricional ${ }^{2,3}$.

Las técnicas de imagen de elección para el diagnóstico pueden ser el tránsito intestinal baritado, el ultrasonido endoscópico, el angioTAC ${ }^{5}$ y la ecografía $^{2}$, de utilidad en AP.

\section{CASO CLÍNICO}

Se trata de un varón de 22 años, asténico, álgido y delgado, que acude a nuestra consulta del centro de salud de forma repetida en el último mes, por dolor epigástrico punzante asociado a náuseas y vómitos postprandiales que alivian el dolor. Los episodios se presentan desde hace 7 años, motivo por el que ha consultado en los servicios de urgencias en múltiples ocasiones, sin que se le haya encontrado nunca patología aguda.

Ingresó hace 3 años por uno de estos episodios, realizándole gastroscopía con biopsia gástrica que resultó positiva para $\mathrm{H}$. pylori; cumplió tratamiento erradicador con triple terapia actual (omeprazol, amoxicilina y claritromicina), con posterior test de aliento negativo para infección por $\mathrm{H}$. pylori. Por persistencia de los síntomas se instauró tratamiento con antieméticos y múltiples inhibidores de la bomba de protones, sin respuesta a largo plazo. En su ingreso fue valorado por el servicio de Salud Mental, ya que persistía la sintomatología de dolor agudo que cedía con los vómitos, siendo diagnosticado de síndrome ansioso-depresivo con mal seguimiento e incumplimiento terapéutico. No presentaba alergias a alimentos ni a fármacos. Actualmente, el dolor cedía en posición genupectoral y con el consumo de cannabis.

A la exploración nos encontramos con un paciente con constantes estables, con un IMC de 17,7. El abdomen está excavado, timpánico, blando y levemente doloroso a la palpación profunda en epigastrio, sin que se palpen masas, adenopatías ni megalias.

La analítica no presentaba alteraciones; ausencia de anemia, marcadores de respuesta inflamatoria negativos, sin alteraciones a nivel hepático ni alteraciones electrolíticas, proteínas totales $5,8 \mathrm{~g} / \mathrm{dl}$ (valor normal de 6,4 a 8,3 g/dL). En la radiografía de abdomen no hay signos de obstrucción intestinal.

Se realizó en nuestra consulta, una ecografía abdominal total en decúbito supino, sin evidencia de hallazgos relevantes, por lo cual decidimos consultar con el servicio de Digestivo, donde, tras ser valorado, se solicitó un tránsito intestinal baritado, con retraso de más de 8 horas por lentitud de vaciamiento gástrico por disminución del calibre en la $3^{\text {a }}$ porción duodenal de forma permanente, sugerente de compresión extrínseca, que no impedía el paso distal de contraste, todo ello compatible con una compresión vascular (figura 1). Ante este hallazgo, se decide realización de angioTAC de abdomen, con el resultado de una pinza vascular que producía compresión del duodeno en su $3^{\mathrm{a}}$ porción. La pinza mesentérica afectaba al duodeno y a la vena renal izquierda, produciendo compresión y dilatación pre y postcompresión (figura 2).

\section{DISCUSIÓN}

El paciente presentaba una clínica de obstrucción intestinal alta de forma crónica, que se iniciaba con dolor epigástrico y posteriormente náuseas y vómitos postprandiales, llevando al paciente a un IMC bajo. Estos casos son demandantes frecuentes de consulta tanto en AP como en el SU, lo que lleva a confundirlos con patologías psicógenas (ansiedad) o psiquiátricas (trastornos de la conducta alimentaria).

El síndrome de Wilkie o SAMS es un reto diagnóstico para nosotros. Se debe sospechar en aquellos pacientes que presentan una pérdida de peso no justificada, acompañada de dolor abdominal de 


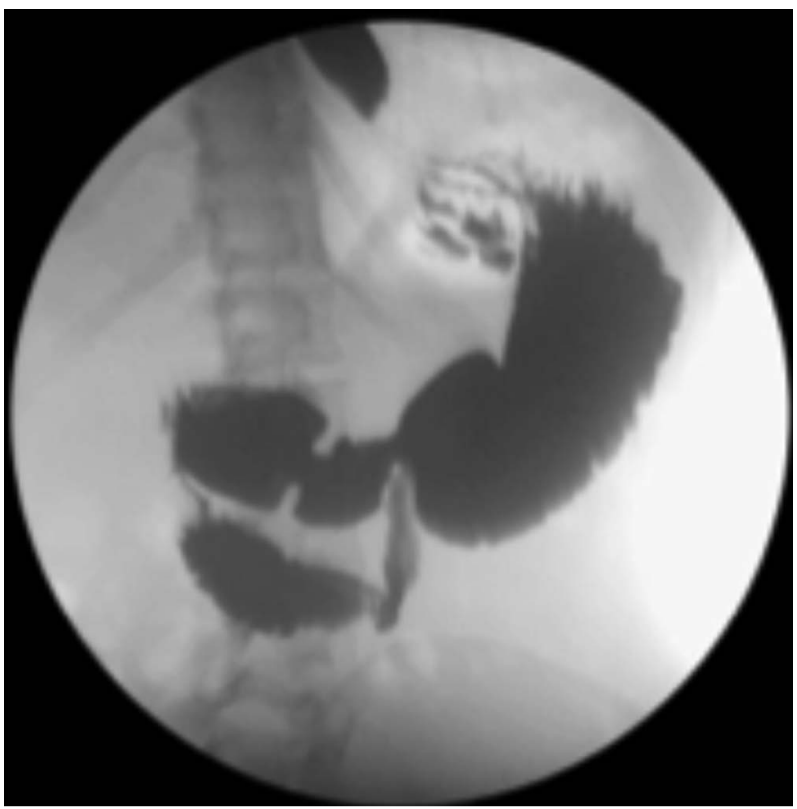

Figura 1. Tránsito intestinal con obstrucción parcial de la $3^{\mathrm{a}}$ porción duodenal

predominio epigástrico asociado a episodios de náuseas, vómitos, sensación de plenitud gástrica y presencia de postura genupectoral en el momento de dolor.

Es una patología de adultos jóvenes, entre los 10 y los 30 años, de predominio en sexo femenino ${ }^{6}$ por causas adquiridas de disminución de la grasa peritoneal debida a pérdida brusca de peso y a la reducción del ángulo aorto-mesentérico. Podemos clasificarlos en 5 grandes grupos de riesgo:

- Síndromes consuntivos (mala absorción intestinal, pacientes oncológicos, grandes quemados).

- Trastornos de la alimentación (anorexia nerviosa).

- Traumatismos severos (TCE - politraumatismos).

- Pacientes con secuelas de cirugía ortopédica de columna vertebral (escoliosis, deformidades óseas).

Congénitas, que incluyen ligamento de Treitz corto y nacimiento anormalmente bajo de la mesentérica superior o presencia de deformidades de la columna vertebral ${ }^{4}$.

Dentro de las técnicas para el diagnóstico se encuentran el transito intestinal baritado, el ultrasoni-

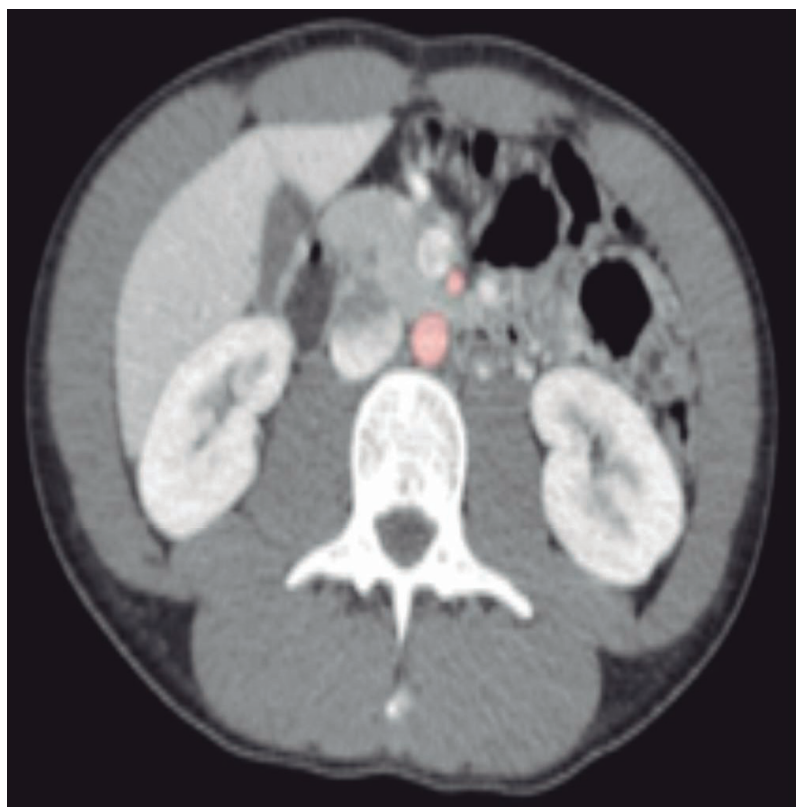

Figura 2. AngioTAC abdominal con Aorta y Mesentérica superior (rojo) comprimiendo la $3^{\text {a }}$ porción del duodeno

do endoscópico, el angioTAC 5 o la ecografía (la cual es útil en AP). Se debe realizar medición del ángulo aorto-mesentérico con el paciente en posición de decúbito lateral; en un corte sagital, dicho ángulo debe ser superior a $25^{\circ}$, normalmente entre $38^{\circ}$ y $65^{\circ 2}$.

En el diagnóstico diferencial se incluyen otras causas de dolor abdominal y obstrucción intestinal como la cetoacidosis diabética, la isquemia mesentérica, intolerancias alimentarias, esclerodermia, enfermedades vasculares del colágeno, pancreatitis, colelitiasis, megaduodeno, bezoares, dispepsia y reflujo ${ }^{4}$.

El manejo médico consiste en la corrección del posible trastorno hidroelectrolítico, la descompresión gástrica con sonda y la nutrición enteral, con una dieta hipercalórica fraccionada y en algunas ocasiones nutrición parenteral, ya que la distribución de la grasa corporal favorece la corrección del ángulo aorto-mesentérico ${ }^{2,3,6}$.

Nuestro objetivo en AP es detectar el trastorno precozmente y garantizar un adecuado aporte nutricional, tratando de lograr índices de masa corporal de normopeso a los pacientes que se encuentren dentro de los grupos de riesgo con dietas fraccionadas hipercalóricas asociado a inhibidores de bomba de protones (IBP).

En última instancia, se optara por el tratamiento quirúrgico en aquellos casos en los que el tratamien- 
to médico ha fracasado ${ }^{4}$. En pacientes crónicos puede darse el fracaso terapéutico al tratamiento médico, lo cual conlleva una mayor prevalencia de úlceras duodenales que en la población general, en un $45 \%$, asociando pérdida de peso con IMC menores a 18, lo que puede llevar a la necesidad de corrección quirúrgica como decisión final|,5,8. Se han propuesto varias soluciones quirúrgicas; la sección duodenal de la $4^{a}$ porción y colocación delante de los vasos, con anastomosis duodenoyeyunal latero-lateral, es de elección, con una tasa de éxito superior al $90 \%$. La gastroyeyunostomía y la división del ligamento de Treitz (operación de Strong) es la técnica de elección en los problemas gastrointestinales congénitos ${ }^{3-5}$.

\section{BIBLIOGRAFÍA}

1. Kendall LJ, Moreira EM. Evaluation of de adult with abdominal pain in the emergency department. Enero $30 \mathrm{de}$ 2014. Disponible en: http:// www.uptodate.com.

2. Scovell S, Hamdan A. Superior mesenteric artery syndrome. Actualizado Feb 2014. Disponible en: http://www.uptodate.com
3. Fernández López MT, López Otero MJ, Bardasco Alonso ML, Álvarez Vázquez P, Rivero Luis MT, López Barros G. Síndrome de Wilkie: a propósito de un caso. Nutr Hosp. 2011;26(3):646-649.

4. Saá Álvarez R, Santidrián Martínez, JI, Oleagoitia Cilaurre JM, Calle Baraja M. Síndrome de Wilkie, una entidad singular. Casos clínicos de cirugía general. Septiembre - Diciembre 2010;2(3):12-4. Disponible en: http://www.aecirujanos. es/revista cirugia casos clinicos/revista.php [consulta 2403-2014]

5. Castaño Llano R, Chams Anturi A, Arango Vargas P, García Valencia A. Síndrome de la arteria mesentérica superior o síndrome de Wilkie. Rev Col Gastroenterol. 2009;24(2):2009.

6. Irastorza Terradillos I, Álvarez Abad I, Santidrián Martinez JI, Vitoria Cormenzana JC. Dolor abdominal recurrente por síndrome de la arteria mesentérica superior. Anales de Pediatria (Barc). 2010;73(3):150 -1.

7. Gerasimidis T, George F. Wilkie syndrome. Dig Surg. 2009; 26(3):213-4.

8. Li J, Chousleb E, Hidalgo J, Patel S, Szomstein S, Rosenthal RJ. Laparoscopic Roux-en-Y duodenojejunal bypass for superior mesenteric artery syndrome: case reports and review of the literature. Surg Laparosc Endosc Percutan Tech. 2011;21(6):e344-7. 